\title{
Detectors and Focal Plane Modules for Weather Instruments
}

\author{
A.I. D’Souza, E. Robinson, S. Masterjohn, V. Khalap \\ DRS Sensors \& Targeting Systems, 10600 Valley View St., Cypress, CA 90630 \\ S. Bhargava, E. Rangel \\ Teledyne Imaging Systems, Camarillo, CA \\ S. Babu \\ NASA Goddard Space Flight Center, 8800 Greenbelt Rd, Greenbelt, MD 20771 \\ D. S. Smith \\ Harris Semiconductor, 1919 W. Cook Rd., Ft. Wayne, IN 46818
}

\begin{abstract}
Weather satellite instruments require detectors with a variety of wavelengths ranging from the visible to VLWIR. The Cross-track infrared Sounder (CrIS) is a Polar Orbiting interferometric sensor that measures earth radiances at high spectral resolution, using the data to provide pressure, temperature and moisture profiles of the atmosphere. The pressure, temperature and moisture sounding data are used in weather prediction models that track storms, predict levels of precipitation etc. The CrIS instrument contains SWIR $\left(\lambda_{c} \sim 5 \mu \mathrm{m}\right.$ at $\left.98 \mathrm{~K}\right)$, MWIR $\left(\lambda_{\mathrm{c}} \sim 9 \mu \mathrm{m}\right.$ at $\left.98 \mathrm{~K}\right)$ and LWIRs $\left(\lambda_{c} \sim\right.$ $15.4 \mu \mathrm{m}$ at $81 \mathrm{~K}$ ) bands in three Focal Plane Array Assemblies (FPAAs).

CrIS detectors are $850 \mu \mathrm{m}$ diameter detectors with each FPAA consisting of nine photovoltaic detectors arranged in a $3 \mathrm{x}$ 3 pattern. Molecular beam epitaxy (MBE)-grown $\mathrm{Hg}_{1-\mathrm{x}} \mathrm{Cd}_{\mathrm{x}} \mathrm{Te}$ material are used for the detectors fabricated in a modified Double Layer Planar Heterostructure (DLPH) architecture. Each detector has an accompanying cold preamplifier. SWIR and MWIR FPAAs operate at $98 \mathrm{~K}$ and the LWIR FPAA at $81 \mathrm{~K}$, permitting the use of passive radiators to cool the detectors. $\mathrm{D}^{*}$ requirements at peak $14.01 \mu \mathrm{m}$ wavelength are $\geq 5.0 \mathrm{E}+10$ Jones for LWIR, $\geq 7.5 \mathrm{E}+10$ Jones at $8.26 \mu \mathrm{m}$ for MWIR and $\geq 3.0 \mathrm{E}+11$ Jones at peak $4.64 \mu \mathrm{m}$ wavelength for SWIR. All FPAAs exceeded the D* requirements. Measured mean values for the nine photodiodes in each of the LWIR, MWIR and SWIR FPAAs are D* $=5.3 \times 10^{10} \mathrm{~cm}$ $\mathrm{Hz}^{1 / 2} / \mathrm{W}$ at $14.0 \mu \mathrm{m}, 9.6 \times 10^{10} \mathrm{~cm}-\mathrm{Hz}^{1 / 2} / \mathrm{W}$ at $8.0 \mu \mathrm{m}$ and $3.4 \times 10^{11} \mathrm{~cm}-\mathrm{Hz}^{1 / 2} / \mathrm{W}$ at $4.64 \mu \mathrm{m}$.
\end{abstract}

\subsection{Introduction}

CrIS is a three-spectral-band Fourier Transform Infrared (FTIR) spectrometer. CrIS employs $850-\mu m$-diameter DLPH Lateral Collection Dectector (LCD) architecture photovoltaic (PV) detectors without ROICs, but each detector is coupled to a separate cold JFET preamplifier and associated passive electronic elements. The detector/amplifier chain requires high performance and linear photoresponse. PV mercury cadmium telluride ( $\mathrm{HgCdTe})$ detectors coupled with the right amplifier provide near-theoretical background-limited infrared performance (BLIP) and the required linear response and are therefore desirable for interferometric infrared sounders. CrIS instrument uses FPAAs for the three spectral bands: SWIR, MWIR, and LWIR. Each FPAA comprises nine separate detectors arranged in a 3 x 3 pattern.

Greater than 100 CrIS size detectors are fabricated on each wafer that also includes Process Evaluation Chips (PECs) with variable detectors. PECs are utilized to determine the cutoff wavelength and quantum efficiency $(\mathrm{QE})$ range for the processed wafer. Dark I-V measurements are collected for every CrIS size detector and those passing dark current and dynamic impedance screens are inserted into Leadless Chip Carriers (LCCs) and evaluated QE and noise. Dark noise for the MWIR and LWIR detectors and under flux conditions for all three colors. From the QE vs wavelength and photo noise measured at the program average operating flux, D* as a function of wavelength is calculated. Detectors that meet CrIS program requirements are used in the Flight FPAAs. This paper outlines the data collected from CrIS detectors and all three FPAAs (SWIR, MWIR \& LWIR). Emphasis is on the LWIR band since it is the most challenging considering it is $15.4 \mu \mathrm{m}$ cutoff at $81 \mathrm{~K}$. 


\subsection{Requirements}

The CrIS spectral bands and operating temperatures of interest determine the HgCdTe material growth parameters. Table I is a listing of the baseline material parameters and the flowdown detector performance required at the operating temperature for each wavelength detector fabricated in that material.

Table I. CrIS detector performance requirements

\begin{tabular}{|c|c|c|c|c|}
\hline Parameter & Units & SWIR & MWIR & LWIR \\
\hline Spectral Band & $\mu \mathrm{m}$ & $3.92-4.64$ & $5.71-8.26$ & $9.13-15.38$ \\
\hline Operating T & $\mathrm{K}$ & 98 & 98 & 81 \\
\hline Detector architecture & & $\mathrm{p}$-on-n & p-on-n & p-on-n \\
& & DLPH & DLPH & DLPH \\
\hline Cutoff wavelength $\lambda_{\mathrm{c}}$ & $\mu \mathrm{m}$ & $\geq 4.96$ & $\geq 8.92$ & $\geq 15.4$ \\
\hline Peak wavelength $\lambda_{\mathrm{p}}$ & $\mu \mathrm{m}$ & 4.64 & 8.26 & 14.01 \\
\hline $\mathrm{R}_{\mathrm{d}} @ \mathrm{~V}_{\mathrm{d}}=-0.1 \mathrm{~V}$ & $\Omega$ & $\geq 5.0 \times 10^{9}$ & $\geq 5.0 \times 10^{5}$ & $\geq 1.0 \times 10^{4}$ \\
\hline $\mathrm{I}_{\mathrm{d}} @ \mathrm{~V}_{\mathrm{d}}=-0.1 \mathrm{~V}$ & $\mathrm{Amps}$ & $\leq 1.7 \times 10^{-10}$ & $\leq 6.0 \times 10^{-7}$ & $\leq 7.0 \times 10^{-5}$ \\
\hline AR-coated QE & & $\geq 0.75$ & $\geq 0.72$ & $\geq 0.6$ \\
\hline Detector diameter & $\mu \mathrm{m}$ & $890 \pm 30$ & $870 \pm 20$ & $865 \pm 20$ \\
\hline
\end{tabular}

\subsection{Detector Technology}

The baseline detector architecture for all three CrIS bands is a p-on-n double layer planar heterostructure (DLPH) diode fabricated by Teledyne Imaging Systems. A DLPH device cross section schematic is shown in Figure 1. Benefits of the $\mathrm{DLPH}^{1-7}$ architecture, include a reduction in surface generation-recombination and tunneling currents, and an increase in total dose radiation hardness, both of which are essential detector attributes for remote sensing applications. Those benefits are realized by incorporating a buried narrow-bandgap active layer in the DLPH architecture. MBE is used to grow the appropriate bandgap n-type $\mathrm{Hg}_{1-\mathrm{x}} \mathrm{Cd}_{\mathrm{x}} \mathrm{Te}$ on lattice matched $\mathrm{CdZnTe}$. $\mathrm{HgCdTe}$ material is grown with wider bandgap cap layers, the subsequent detector architecture translating into the DLPH architecture. $850 \mu \mathrm{m}$ diameter detectors are manufactured using the lateral collection diode (LCD) architecture ${ }^{8}$ to reduce the probability of a performance degrading defect ${ }^{9}$ intersecting the detector $\mathrm{p} / \mathrm{n}$ junction. A modified detector architecture has been implemented to improve bake stability of the detector architecture. ${ }^{10}$

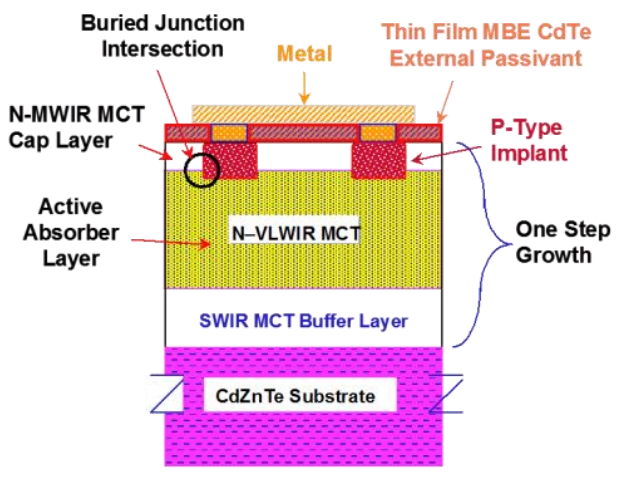

Figure 1: Cross section of an MBE grown DLPH detector architecture

\subsection{Detector Evaluation}

Detectors fabricated on a wafer are wirebonded in a carrier and dark I-V measurements are conducted at the $98 \mathrm{~K}$ for SWIR and MWIR and $81 \mathrm{~K}$ for LWIR operating temperatures. The magnitude of the current and shape of the I-V 
characteristics serves as a screen to pick detectors that will be inserted into Leadless Chip Carriers (LCCs). Dark I-V curves from a sample LWIR wafer are plotted in Figure 2. Although there are some leaky and shorted detectors, a remarkable $\sim 60 \%$ of the detectors on the wafer meet the CrIS requirements with a number of the detectors diffusion limited out to the $-200 \mathrm{mV}$ measured. Some of the candidate detector selected from the wafer dark I-V measurement are mounted into LCCs for further evaluation regarding suitability of the detectors for insertion into flight modules. Detector measurements in LCCs dark and multiple flux conditions for I-V characteristics and linearity, noise under dark and illuminated conditions, spectral response and quantum efficiency (QE) performance at the specified operating temperature. Dark I-V from $-200 \mathrm{mV}$ to $150 \mathrm{mV}$, I-V while illuminated under ten flux values to extract non-linearity and noise spectral density at $-100 \mathrm{mV}$ and $-150 \mathrm{mV}$. Photo noise spectral density measurements are at the CrIS instrument flux values in the mid $10^{17}$ photon/ $\mathrm{cm}^{2} / \mathrm{s}$ for LWIR and mid $10^{16}$ photon $/ \mathrm{cm}^{2} / \mathrm{s}$ for MWIR detectors.

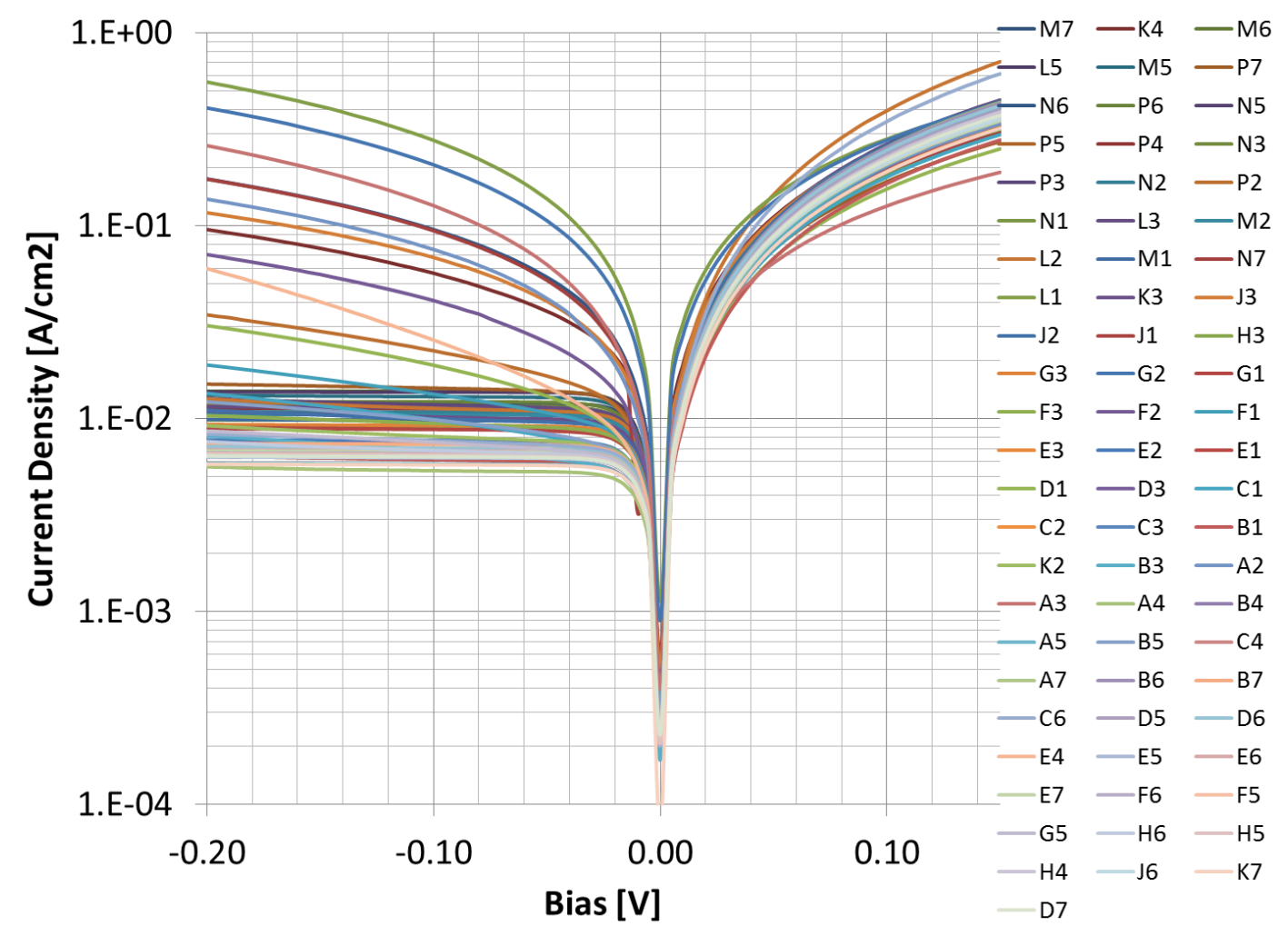

Figure 2. Sample dark I-V curves from an LWIR wafer at $81 \mathrm{~K}$

A commercial Fourier transform infrared spectrometer is used to obtain the spectral response at $81 \mathrm{~K}$ for all the LWIR photodiodes. All measurements are made at the temperature and bias at which the photodiodes will operate within the CrIS instrument. For the LWIR photodiodes, absolute QE is measured at $\lambda=10.6 \mu \mathrm{m}$ using a narrow band filter. Narrow band filters centered at $\lambda=7.4 \mu \mathrm{m}$ and $\lambda=4.0 \mu \mathrm{m}$ are used for the MWIR and SWIR photodiodes QE measurements. The QE and spectral cut-off are traceable to NIST. The quantum efficiency using a narrow band filter is combined with the spectral response curve to obtain the QE versus wavelength curve as displayed in Figure 3 for a representative LWIR photodiode. QE as a function of wavelength is a principal specification that has to be met for a photodiode to be utilized in the CrIS instrument. The specification QE is also displayed in Figure 3 as a dashed red line. As can be seen from the figure the QE is high. Fringes seen in the data are a result of etalon effects in the anti-reflection coating and the detector. Modeling of QE vs wavelength have been described previously. ${ }^{11}$ 


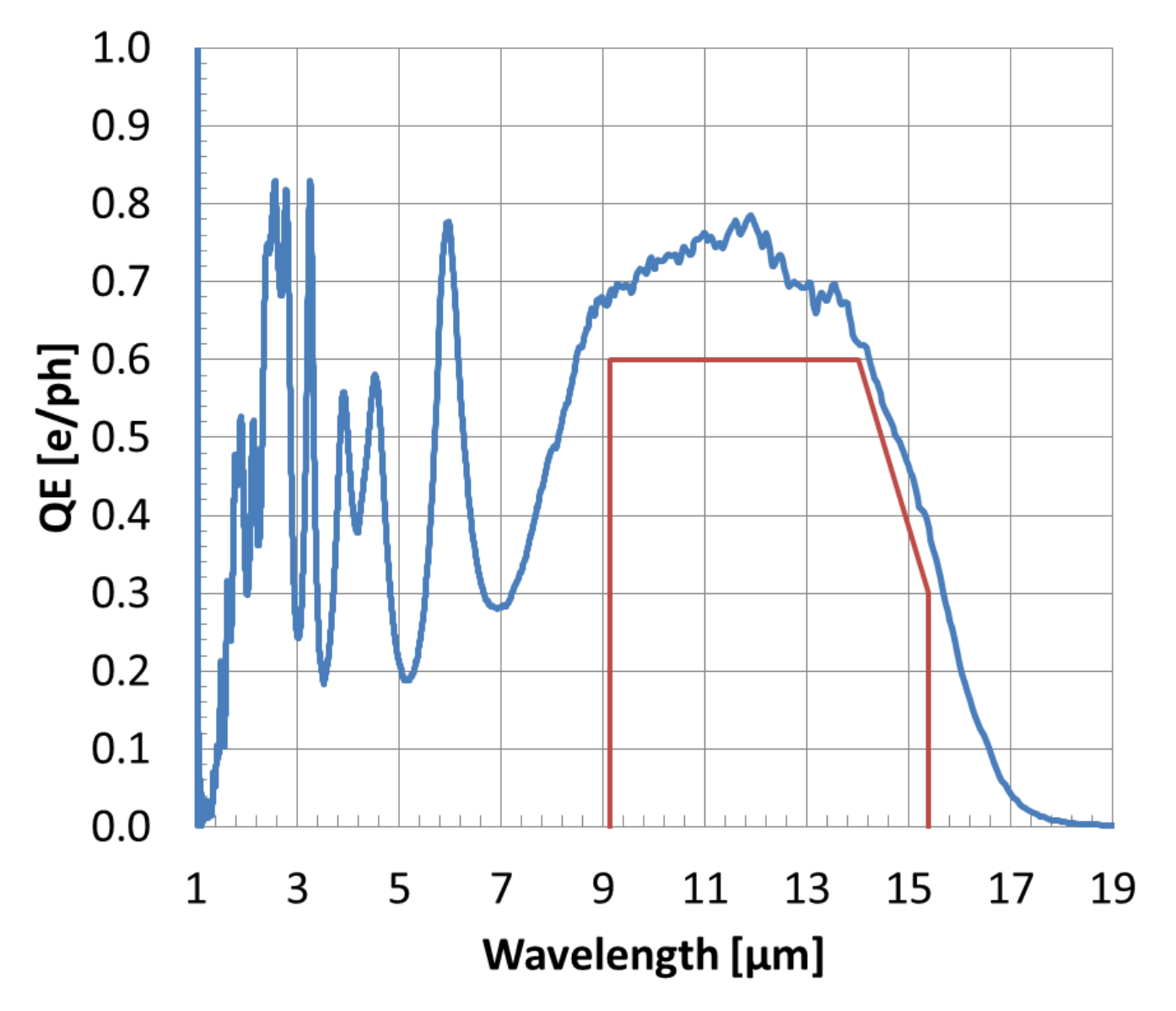

Figure 3. Quantum efficiency versus wavelength for a LWIR $\left[\lambda_{c}(81 \mathrm{~K}) \sim 15.4 \mu \mathrm{m}\right]$ photodiode

$\mathrm{I}-\mathrm{V}$ and noise is measured ${ }^{12,13}$ for all photodiodes in each LCC at the operating temperature, i.e. LWIR are measured at $81 \mathrm{~K}$, whereas MWIR and SWIR photodiodes are measured at $98 \mathrm{~K}$. Figure $4 \mathrm{a}$ is the noise spectral density curves in the dark and under illuminated conditions for the same diode shown in Figure 3. Noise spikes are reflective of pickup from the electronics and cables and not from the detector. The detector is shot noise limited down to $\sim 500 \mathrm{~Hz}$. Figure $4 \mathrm{~b}$ is the calculated $\mathrm{D}^{*}$ as a function of wavelength using the QE from Figure 3 and the noise from Figure 4a. The line at $14 \mu \mathrm{m}$ extending up to $5 \times 10^{10}$ Jones is the specification. The $\mathrm{D}^{*}$ exceeds the specification with margin. These are the photodiodes that are selected for placement in the FPAA. Similar measurements and calculations are made for the MWIR photodiodes. SWIR photodiodes at $98 \mathrm{~K}$ have calculated white noise values in the low $10^{-15} \mathrm{~A} / \mathrm{Hz}^{1 / 2}$ range. This noise level is impossible to measure consistently and accurately using the noise measurement station and commercial amplifiers such as the Ithaco 1211 that are run at room temperature. and $\Phi=1.8 \times 10^{15} \mathrm{ph} / \mathrm{cm}^{2} / \mathrm{s}$ respectively. Similar procedures are followed for the selection of MWIR and SWIR photodiodes prior to their selection for placement into FPAAs.

Noise limits the sensitivity of the CrIS instrument. Noise current spectral density has been measured on a number of 850 $\mu \mathrm{m}$ diameter CrIS MWIR and LWIR detectors under dark and illuminated conditions. Detectors are held at $-100 \mathrm{mV}$ bias and MWIR detector measurements are at $98 \mathrm{~K}$ and LWIR at $81 \mathrm{~K}$, the CrIS instrument operating temperatures. Noise comparisons are made between detectors fabricated within the past year for the CrIS J2 instrument to detectors fabricated in 2002 for the CrIS J1 instrument. The performance parameter compared is the Tobin coefficient $\alpha,{ }^{14}$ defined as the ratio of the noise at $1 \mathrm{~Hz}$ to the current at the bias the noise is measured. Previous measurements ${ }^{12}$ had MWIR detectors Tobin coefficient in the dark, $\alpha_{\mathrm{MW}-\mathrm{d}}=i_{n} / I_{d}=1.93 \pm 0.63 \times 10^{-4}$. Measurements on forty nine MWIR detectors under illuminated conditions at $98 \mathrm{~K}$ and $-60 \mathrm{mV}$ bias resulted in $\alpha_{\mathrm{MW}-\mathrm{P}}=i_{n} / I_{p h}=4.16 \pm 1.69 \times 10^{-6}$. For LWIR detectors, the best performers had dark $\alpha_{\mathrm{LW}-\mathrm{d}} \sim 5-7 \times 10^{-6}$, near the photo-induced ratio. However, the majority of LWIR detectors 
had dark $\alpha_{\mathrm{LW}-\mathrm{d}} \sim 2 \times 10^{-5}$. A summary of the Tobin coefficients extracted from the noise spectral density curves and the standard deviation for the detectors are listed in Table I. MWIR dark $\alpha_{\mathrm{MW}-\mathrm{d}}$ are an order of magnitude lower compared to the detectors manufactured a decade earlier. MWIR photo $\alpha_{\text {MW-P }}$ are $2-3 \mathrm{X}$ lower, LWIR dark $\alpha_{\mathrm{LW}-\mathrm{d}}$ are $\sim 2 \mathrm{X}$ lower and LWIR photo $\alpha_{\mathrm{LW}-\mathrm{P}}$ are $\sim 4 \mathrm{X}$ lower. The best $\alpha_{\mathrm{LW}-\mathrm{d}}$ are in the $4-7 \times 10^{-6}$ range.
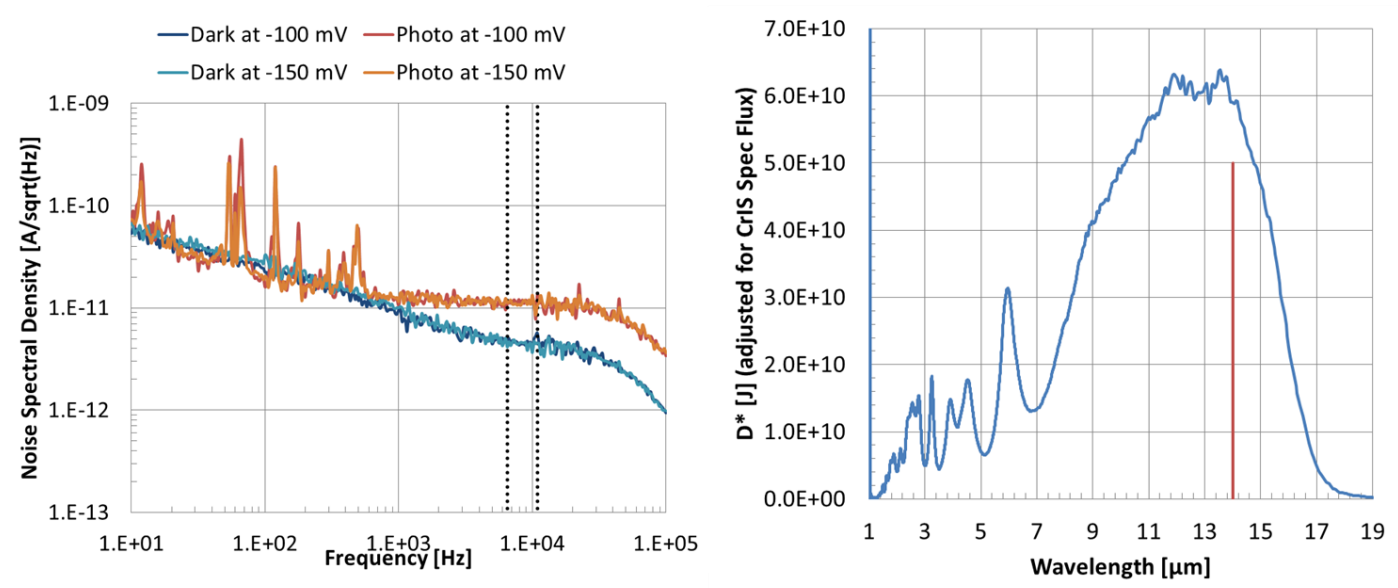

Figure 4a and 4b. Dark and illuminated noise spectral density and $\mathrm{D}^{*}$ for representative LWIR photodiode at $81 \mathrm{~K}$.

Table I. Tobin values summary under dark and illuminated conditions

\begin{tabular}{|c|c|c|c|}
\hline \multicolumn{2}{|c|}{ MWIR $\alpha_{\text {dark }}$} & \multicolumn{2}{c|}{ MWIR $\alpha_{\text {Photo }}$} \\
\hline Average & $1.73 \mathrm{E}-05$ & Average & $1.63 \mathrm{E}-06$ \\
\hline$\sigma$ & $5.49 \mathrm{E}-06$ & $\sigma$ & $3.47 \mathrm{E}-07$ \\
\hline Number & 25 & Number & 25 \\
\hline
\end{tabular}

\begin{tabular}{|c|c|c|c|}
\hline \multicolumn{2}{|c|}{$L W I R \alpha_{\text {dark }}$} & \multicolumn{2}{c|}{ LWIR $\alpha_{\text {photo }}$} \\
\hline Average & $1.10 E-05$ & Average & $1.15 E-06$ \\
\hline$\sigma$ & $5.29 E-06$ & $\sigma$ & $7.33 E-07$ \\
\hline Number & 27 & Number & 27 \\
\hline
\end{tabular}

I-V curves are acquired at ten different flux values encompassing the CrIS FTIR instrument flux ranges of $2.96 \times 10^{17}$ $\mathrm{ph} / \mathrm{cm}^{2} / \mathrm{s}$ to $4.04 \times 10^{17} \mathrm{ph} / \mathrm{cm}^{2} / \mathrm{s}$ for the LWIR and $5.11 \times 10^{16} \mathrm{ph} / \mathrm{cm}^{2} / \mathrm{s}$ to $7.36 \times 10^{16} \mathrm{ph} / \mathrm{cm}^{2} / \mathrm{s}$ for the MWIR. Nonlinearity, defined as the maximum deviation of the signal current at any flux from the least squares fit line at all ten points divided by the total current swing have requirements that are quite stringent, $0.15 \%$ for LWIR and $0.16 \%$ for MWIR. Non-linearity is measured as a function of bias and shown in figure 5a are the I-Vs as a function of flux for an MWIR detector and in figure $5 b$ is an example of current as a function of flux. Deviation of the current at each flux from the least squares line fit is listed in Table II. Maximum deviation is the non-linearity for the detector. Detectors that meet all CrIS instrument requirements such as QE vs wavelength, D*, non-linearity are deemed flight quality detectors. 

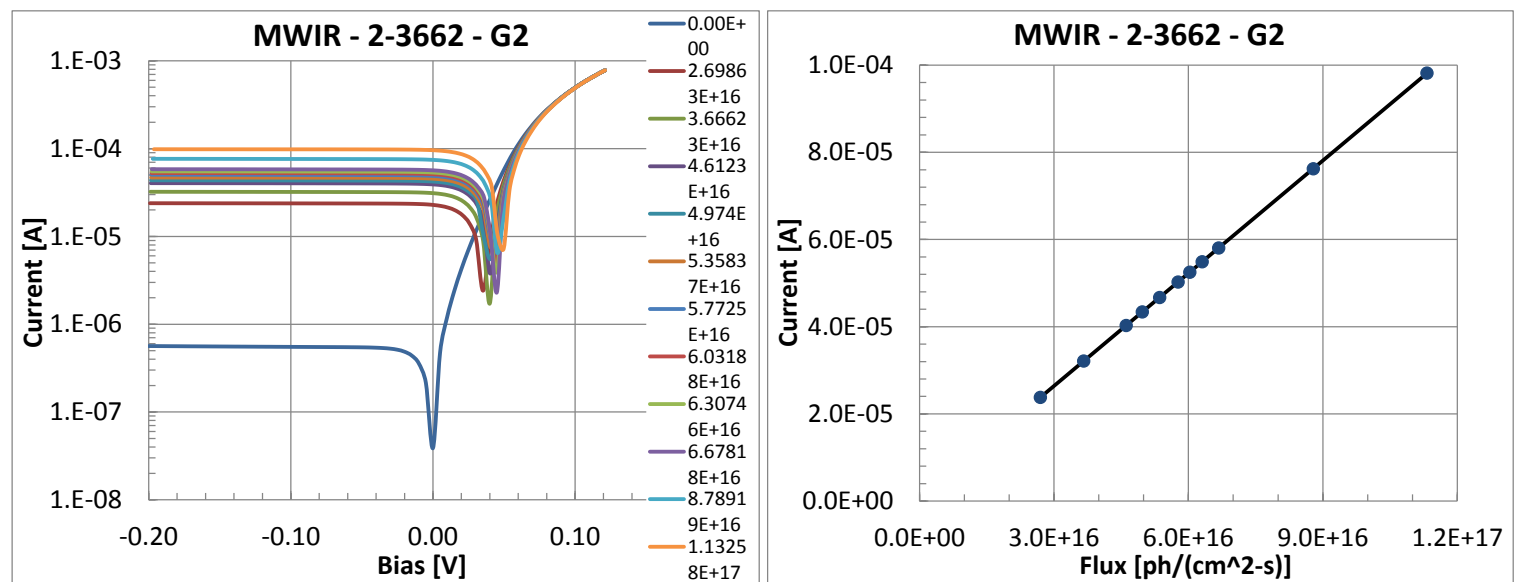

Figure 5. (a) $\mathrm{I}-\mathrm{V}$ as a function of flux and least squares fit to current at $-100 \mathrm{mV}$

Table II. Calculation of non-linearity from the measured and least squares fit in Figure 5

\begin{tabular}{|l|c|c|c|c|c|c|c|c|c|c|c|}
\hline & $2.7 \mathrm{E}+16$ & $3.666 \mathrm{E}+16$ & $4.61 \mathrm{E}+16$ & $4.97 \mathrm{E}+16$ & $5.36 \mathrm{E}+16$ & $5.77 \mathrm{E}+16$ & $6.03 \mathrm{E}+16$ & $6.31 \mathrm{E}+16$ & $6.68 \mathrm{E}+16$ & $8.79 \mathrm{E}+16$ & $1.13 \mathrm{E}+17$ \\
\hline Best line & $2.38 \mathrm{E}-05$ & $3.21 \mathrm{E}-05$ & $4.03 \mathrm{E}-05$ & $4.34 \mathrm{E}-05$ & $4.67 \mathrm{E}-05$ & $5.03 \mathrm{E}-05$ & $5.25 \mathrm{E}-05$ & $5.49 \mathrm{E}-05$ & $5.81 \mathrm{E}-05$ & $7.63 \mathrm{E}-05$ & $9.82 \mathrm{E}-05$ \\
\hline Linearity & $0.04 \%$ & $0.05 \%$ & $0.04 \%$ & $0.08 \%$ & $0.07 \%$ & $0.13 \%$ & $0.09 \%$ & $0.07 \%$ & $0.09 \%$ & $0.13 \%$ & $0.01 \%$ \\
\hline $\max$ & $0.13 \%$ & & & & & & & & & & \\
\hline
\end{tabular}

\subsection{CrIS Focal Plane Array Assembly (FPAA)}

The Flight configuration for the CrIS Detector Preamplifier Module (DPM) consists of three spectrally separate (SWIR, MWIR and LWIR) FPAAs, ${ }^{15,16}$ three signal flex cable assemblies, a warm signal flex cable, vacuum bulk head assembly, and the warm electronics circuit card assemblies (CCAs) as shown in figure 6 . The photodiodes are coupled to a differential J-FET buffered, resistive feedback, transimpedance amplifier (RTIA). The RTIA provides the necessary gain for the circuit, and the J-FET differential buffers provide low noise, current to voltage conversion at constant output impedance. Each FPAA contains a $3 \times 3$ array of nine $850 \mu \mathrm{m}$ diameter photovoltaic detectors, each driving its own RTIA, with their associated cold electronics, detector optics assembly, and two flex cable assemblies with interface connectors. The FPAAs (detector arrays, detector optics assemblies, JFET buffer and feedback resistor portions of the transimpedance preamplifier, and flex cable assemblies) are cooled by the detector cooler module in their final configuration. The cryogenic portions of the DPM (FPAAs, and Signal Flex Cable Assemblies) mate to the ambient temperature portions of the DPM (warm signal flex cable assembly and the ambient temperature portions of the transimpedance amplifier, mounted within the CCAs) through the vacuum bulk head assembly mounted on the detector cooler assembly housing. The FPAA assembly is connected to the warm electronics via a constantan cable and vacuum feedthru hermetic connector. Each FPAA has its own Circuit Card Assembly (CCA) that contains a warm amplifier and the second stage $300 \mathrm{~Hz}$ high pass filter. One warm cable assembly provides the FPAA signals for all three bands. The CCA also provides the biases for the cold JFET pair on the cold electronics Ceramic Multilayer Board (CMLB). 


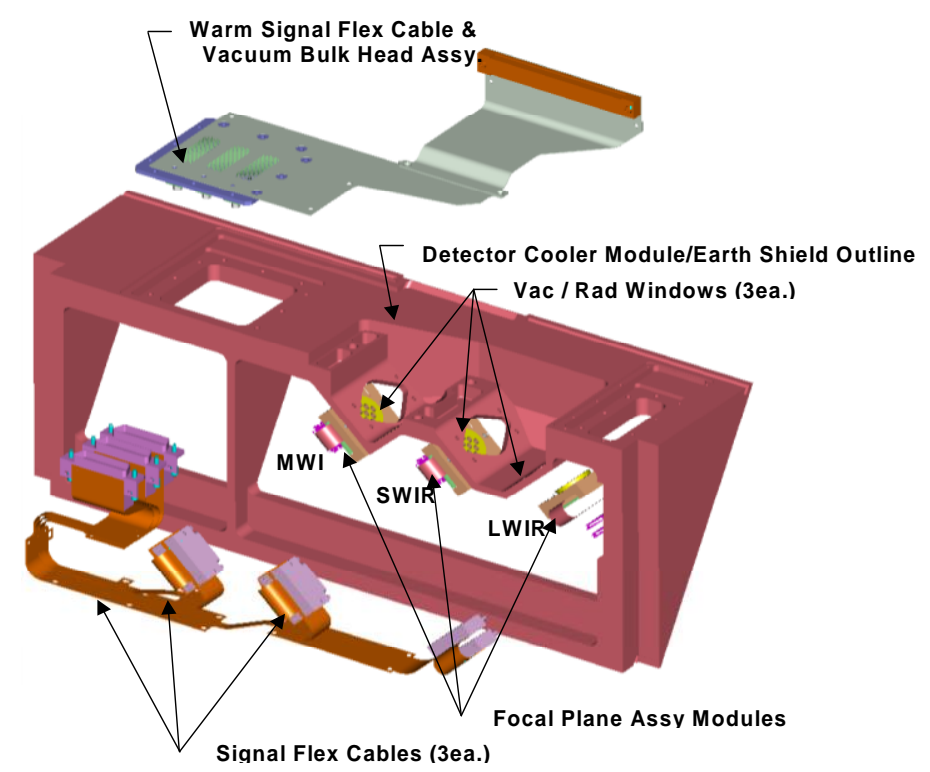

Figure 6. CrIS detector Preamplifier Module (Warm electronics CCAs not shown)

Quantum efficiency versus wavelength and noise spectral density under dark and illuminated conditions are used to determine the nine photodiodes of each color that are integrated in the FPAA. These detectors are placed in a $3 \times 3$ grid array on the base of the module with a placement accuracy of $+/-2 \mu \mathrm{m}$ from FOV to FOV. Placement of the photodiodes into the sapphire carrier is a critical step in the assembly of the FPAA. Careful procedures are followed to prevent degradation in performance through the FPAA mounting and assembly sequence. A picture of an assembled FPAA is shown in figure 7. Since the photodiodes are backside illuminated, the top metal pad that connects the p-type implants that form a single $850 \mu \mathrm{m}$ diameter lateral collection detector is seen in the picture. Also seen are the discrete components (JFETs, resistors etc.) that constitute the preamplifier module.

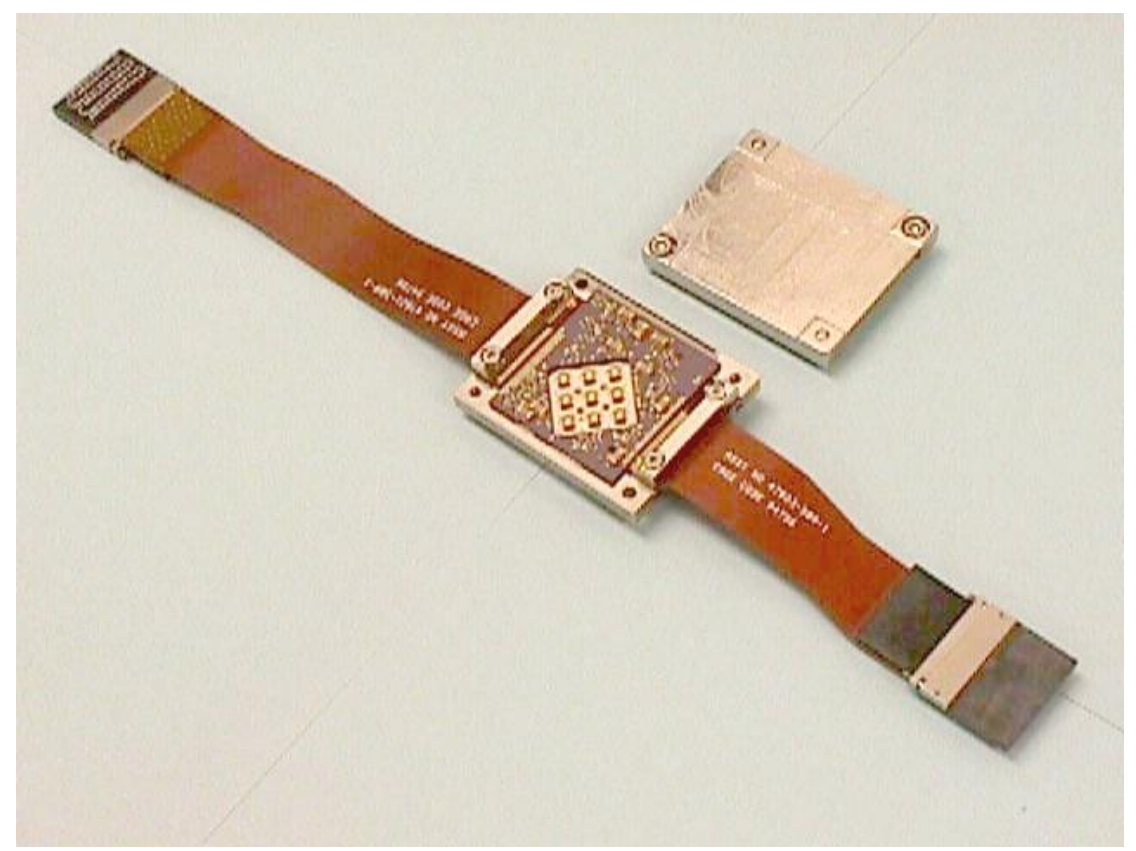

Figure 7. Assembled CrIS LWIR FPAA module

\subsection{FPAA Performance}




\subsection{LWIR FPAA Data}

The LWIR FPAA is cooled to $81 \mathrm{~K}$ and the photodiodes held at $100 \mathrm{mV}$ reverse bias during subsequent responsivity and noise measurements. The reason for holding the photodiodes in reverse bias is two-fold. The impedance of large $\lambda_{\mathrm{c}}(81 \mathrm{~K})$ $\sim 15.4 \mu \mathrm{m}$ photodiodes is quite low at zero bias. A pre-amplifier interfacing with a low impedance detector results in high noise in the pre-amplifier. Also, the injection efficiency is depressed, rendering it impossible to extract the photocurrent efficiently. Consequently, the external quantum efficiency is low. Operating the detector in reverse bias can increase the detector dynamic impedance by two orders of magnitude, resulting in the external quantum efficiency rising to the value of the internal quantum efficiency.

Noise measurements at the nominal operating flux of $\Phi=3.5 \times 10^{17}$ photons $\cdot \mathrm{cm}^{-2} \cdot \mathrm{s}^{-1}$ are made for all the photodiodes in the FPAA. These flux values are achieved using an Electro-Optical Industries (EOI) Black Body mounted external to the dewar. The dewar flux is calibrated using a detector with known QE. For the LWIR FPAA, the electrical band of interest is $6.5-10.95 \mathrm{kHz}$. To obtain maximum performance, the photodiode noise needs to be photon noise dominated within the frequency band of interest. The gain of the pre-amplifier is designed to be maintained constant within the frequency band of interest. There is a one-to-one correspondence between response at a particular wavelength $\lambda_{\mathrm{l}}$ and noise at frequency $\mathrm{f}_{\mathrm{i}}$. To calculate $\mathrm{D}^{*}$ at any wavelength, the quantum efficiency at any wavelength $\eta(\lambda)$, is ratioed to the noise corresponding to that particular wavelength. The other parameters listed in the $\mathrm{D}^{*}$ equation 1 below are then input to calculate $\mathrm{D}^{*}$ as a function of wavelength, $D^{*}(\lambda)$. Figure 8 is a plot of QE and $\mathrm{D}^{*}$ versus $\lambda$ for all the nine photodiodes. Some of the detectors $\mathrm{D}^{*}$ are marginally below $5.0 \times 10^{10} \mathrm{~cm} \mathrm{~Hz}^{1 / 2} / \mathrm{W}$ at $14 \mu \mathrm{m}$. This is purely due to the cutoff wavelength of the detectors being $\sim 15 \mu \mathrm{m}$, marginally shorter than the $15.4 \mu \mathrm{m}$ required. All the detectors were photon shot noise limited in the CrIS band of interest. Peak D* is in the $5.5-6.0 \times 10^{10} \mathrm{~cm} \mathrm{~Hz}^{1 / 2} / \mathrm{W}$ range at $\sim 13.5 \mu \mathrm{m}$. A new module is to be fabricated with slightly longer cutoff wavelength detectors that will insure all the detectors having $\mathrm{D}^{*}$ exceeding $5.0 \times 10^{10} \mathrm{~cm} \mathrm{~Hz}^{1 / 2} / \mathrm{W}$ at $14 \mu \mathrm{m}$.

$$
\boldsymbol{D} *(\lambda)=\frac{\boldsymbol{q} \lambda \boldsymbol{A}_{\operatorname{det}}{ }^{\frac{1}{2}} \eta(\lambda)}{\boldsymbol{h c i _ { n }}(\lambda)}
$$
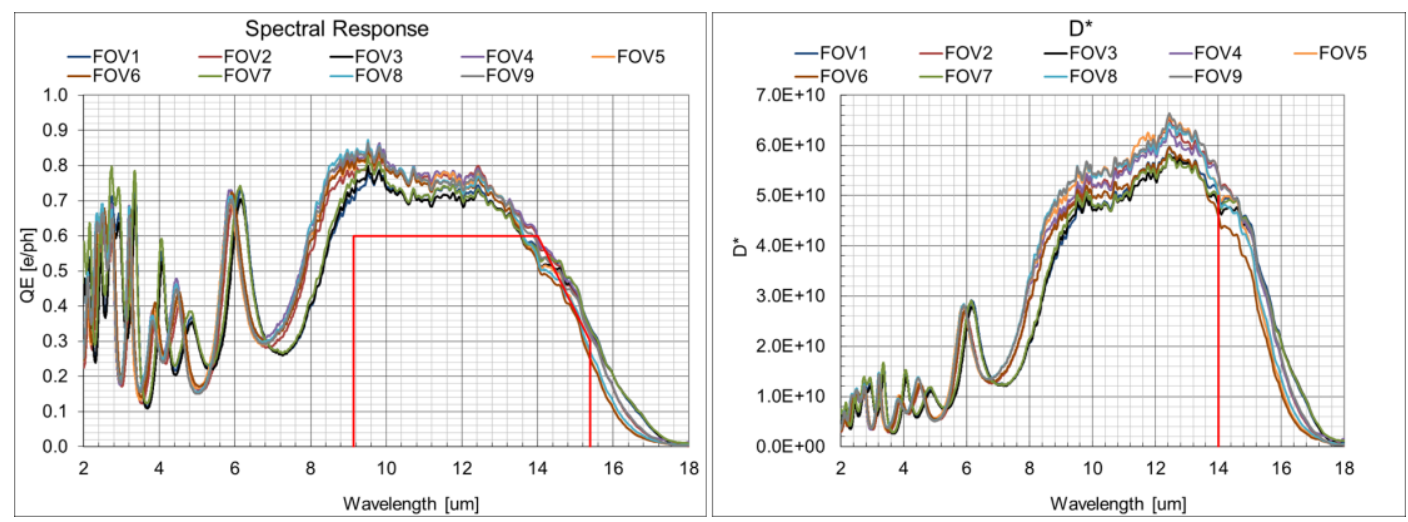

Figure 8. LWIR Spectral D* at $81 \mathrm{~K}$ and nominal flux $\Phi=3.5 \times 10^{17}$ photons $\cdot \mathrm{cm}^{-2} \cdot \mathrm{s}^{-1}$

\subsection{MWIR FPAA Data}

The MWIR FPAA is cooled to $98 \mathrm{~K}$ and the photodiodes are also held at $100 \mathrm{mV}$ reverse bias as in the LWIR FPAA case. Noise measurements are made at the nominal $\Phi=6.2 \times 10^{16}$ photons $\cdot \mathrm{cm}^{-2} \cdot \mathrm{s}^{-1}$. The electrical band of interest is 
12.1-17.5 kHz, corresponding to wavelengths $\lambda=8.26 \mu \mathrm{m}$ down to $\lambda=5.71 \mu \mathrm{m}$. Spectral $\mathrm{QE}$ and $\mathrm{D}^{*}$ as a function of wavelength is plotted in Figure 9. The average $\mathrm{D}^{*}$ at $8.26 \mu \mathrm{m}$ was $9.57 \times 10^{10}+/-4.16 \times 10^{9} \mathrm{~cm}-\mathrm{Hz}^{1 / 2} / \mathrm{W}$, exceeding the specification $\mathrm{D}^{*}=7.5 \times 10^{10} \mathrm{~cm}-\mathrm{Hz}^{1 / 2} / \mathrm{W}$ by $27.6 \%$.
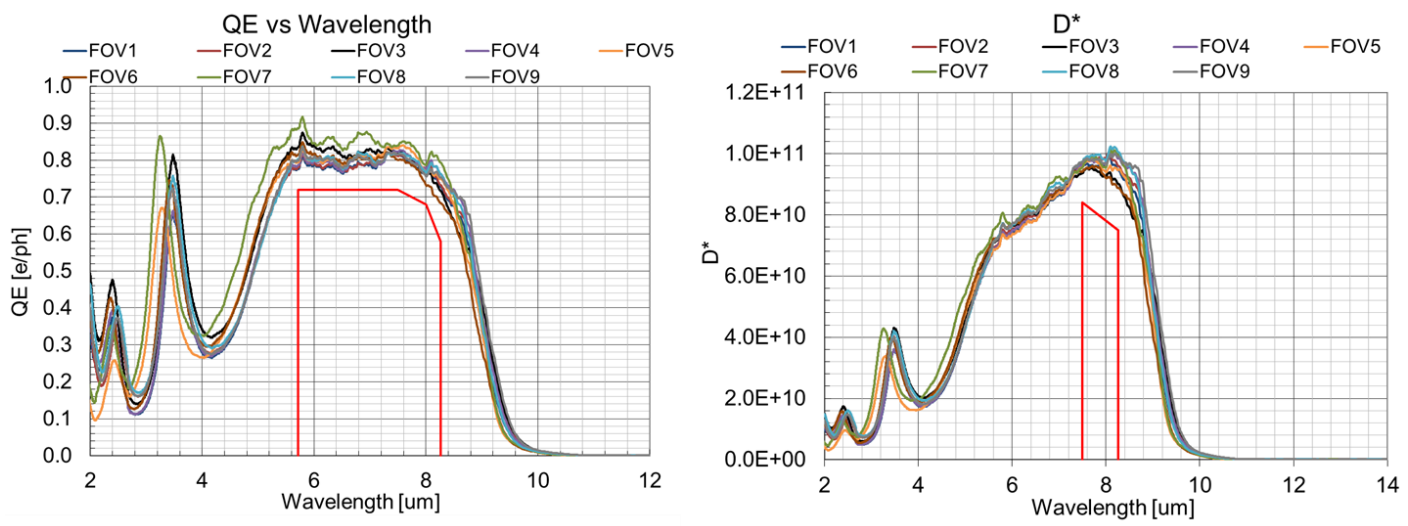

Figure 9. MWIR Spectral D* at $98 \mathrm{~K}$ and nominal flux $\Phi=6.2 \times 10^{16}$ photons $\cdot \mathrm{cm}^{-2} \cdot \mathrm{s}^{-1}$ 


\subsection{SWIR FPAA Data}

The SWIR FPAA is cooled to $98 \mathrm{~K}$ and the detectors are held at $60 \mathrm{mV}$ reverse bias. Noise measurements are made at the nominal $\Phi=1.8 \times 10^{15}$ photons $\cdot \mathrm{cm}^{-2} \cdot \mathrm{s}^{-1}$. The electrical band of interest is $21.5-25.5 \mathrm{kHz}$, corresponding to wavelengths $\lambda=4.64 \mu \mathrm{m}$ down to $\lambda=3.92 \mu \mathrm{m}$. Spectral $\mathrm{D}^{*}$ as a function of wavelength is plotted in Figure 10 . The average $\mathrm{D}^{*}$ at $4.64 \mu \mathrm{m}$ is $3.38 \times 10^{11}+/-1.21 \times 10^{10} \mathrm{~cm}-\mathrm{Hz}^{1 / 2} / \mathrm{W}$, exceeding the specification $\mathrm{D}^{*}=3.0 \times 10^{11} \mathrm{~cm}^{-\mathrm{Hz}} \mathrm{z}^{1 / 2} / \mathrm{W}$ by $12.6 \%$ and close to the BLIP D* of $4.2 \times 10^{11} \mathrm{~cm}-\mathrm{Hz}^{1 / 2} / \mathrm{W}$. The BLIP D* is calculated using a $\mathrm{QE}=1$.
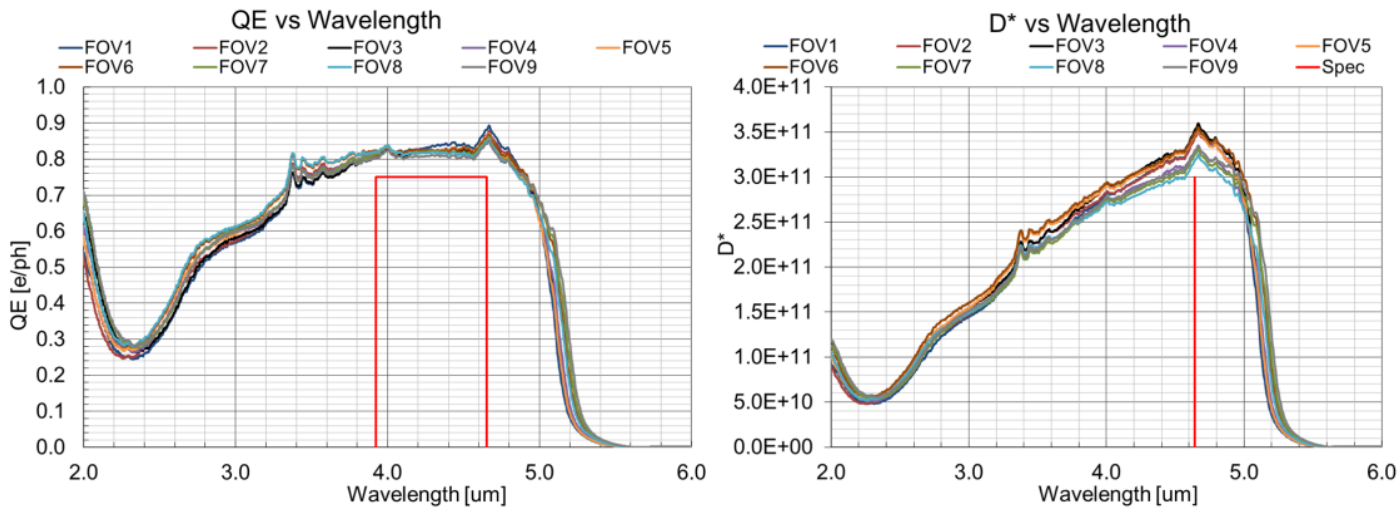

Figure 10. SWIR Spectral D* at $98 \mathrm{~K}$ and nominal flux $\Phi=1.8 \mathrm{Y} \times 10^{15}$ photons $\cdot \mathrm{cm}^{-2} \cdot \mathrm{s}^{-1}$

\subsection{SUMMARY}

The appropriate bandgap n-type $\mathrm{Hg}_{1-\mathrm{x}} \mathrm{Cd}_{\mathrm{x}} \mathrm{Te}$ was grown on lattice-matched CdZnTe. 850- $\mu$ m-diameter photodiodes were manufactured using a Lateral Collection Diode (LCD) architecture. Custom pre-amplifiers are separately designed to interface with the large LWIR and MWIR low impedance photodiodes and with SWIR photodiodes at frequencies up to $25.5 \mathrm{kHz}$. Pre-amplifier gain is maintained constant within the electrical band of interest for each color. The LWIR, MWIR and SWIR photodiodes are operated at $81 \mathrm{~K}, 98 \mathrm{~K}$ and $98 \mathrm{~K}$ respectively. Performance goals are $\mathrm{D}^{*}=5.0 \mathrm{x} 10^{10}$ $\mathrm{cm}-\mathrm{Hz}^{1 / 2} / \mathrm{W}$ at $14.0 \mu \mathrm{m}, 7.5 \times 10^{10} \mathrm{~cm}-\mathrm{Hz}^{1 / 2} / \mathrm{W}$ at $8.26 \mu \mathrm{m}$ and $3.0 \times 10^{11} \mathrm{~cm}-\mathrm{Hz}^{1 / 2} / \mathrm{W}$ at $4.64 \mu \mathrm{m}$. Measured mean values for the nine photodiodes in each of the LWIR, MWIR and SWIR FPAAs are D* $=5.3 \times 10^{10} \mathrm{~cm}^{-\mathrm{Hz}}{ }^{1 / 2} / \mathrm{W}$ at $14.01 \mu \mathrm{m}, 9.6$ $\mathrm{x} 10^{10} \mathrm{~cm}-\mathrm{Hz}^{1 / 2} / \mathrm{W}$ at $8.26 \mu \mathrm{m}$ and $3.1 \times 10^{11} \mathrm{~cm}-H z^{1 / 2} / \mathrm{W}$ at $4.64 \mu \mathrm{m}$. These compare favorably with the BLIP calculated at the nominal flux condition are $\mathrm{D}^{*}=8.36 \times 10^{10} \mathrm{~cm} \mathrm{~Hz}^{1 / 2} / \mathrm{W}$ at $14.01 \mu \mathrm{m}, 1.4 \mathrm{x} 10^{11} \mathrm{~cm}^{-\mathrm{Hz}^{1 / 2}} / \mathrm{W}$ at $8.26 \mu \mathrm{m}$ and $3.4 \mathrm{x}$ $10^{11} \mathrm{~cm}-\mathrm{Hz}^{1 / 2} / \mathrm{W}$ at $4.64 \mu \mathrm{m}$.

\subsection{ACKNOWLEDGEMENTS}

This work was supported by NASA under the CrIS program. However, "The views expressed are those of the author and do not reflect the official policy or position of the U.S. Government." Special thanks to our colleagues at NASA, Harris, Teledyne Imaging System and DRS who have provided outstanding assistance and support.

\subsection{REFERENCES}

1. J.M. Arias, S.H. Shin, J.G. Pasko, R.E. DeWames, E.R. Gertner, J. Appl. Phys. 65, 1747 (1989).

2. J.M. Arias, M. Zandian, J.G. Pasko, S.H. Shin, L.O. Bubulac, R.E. DeWames, W.E. Tennant, J. Appl. Phys. 69, 2143 (1991).

3. J. Bajaj, J.M. Arias, M. Zandian, J.G. Pasko, L.J. Kozlowski, R.E. DeWames, W.E. Tennant, J. Electron. Mater. 24, 1067 (1995).

4. J.M. Arias, J.G. Pasko, M. Zandian, S.H. Shin, G. M. Williams, L.O. Bubulac, R.E. DeWames, W.E. Tennant, Appl. Phys. Lett. 62, 976 (1993). 
5. A.I. D'Souza, L.C. Dawson, E.J. Anderson, A.D. Markum, W.E. Tennant, L.O. Bubulac, M. Zandian, J.G. Pasko, W.V. McLevige, D.D. Edwall, J. Electron. Mater. 26, 656 (1997).

6. J.M. Arias, J.G. Pasko, M. Zandian, S.H. Shin, G.M. Williams, L.O. Bubulac, R.E. DeWames, W.E. Tennant, J. Electron. Mater. 22, 1049 (1993).

7. S.H. Shin, J.M. Arias, M. Zandian, J.G. Pasko, L.O. Bubulac, R.E. DeWames, J. Electron. Mater. 22, 1049 (1993).

8. H. Holloway, J. Appl. Phys., 49, 4264 (1978)

9. P.S. Wijewarnasuriya, M. Zandian, D.B. Young, J. Waldrop, D.D. Edwall, W.V. McLevige, J. Arias, A.I. D'Souza, J. Electron. Mater. 28, 649 (1999).

10. B.J. Rauscher, C. Stahle, R.J. Hill, M. Greenhouse, J. Beletic et al, AIP Advances 2, 021901 (2012).

11. A.I. D'Souza, E. Robinson, M.G. Stapelbroek, P.S. Wijewarnasuriya, J. of Electronic Materials, Vol. 40, No. 8, 1657 (2011).

12. A.I. D’Souza, M.G. Stapelbroek, P.N. Dolan, P.S. Wijewarnasuriya, R.E. DeWames, D.S. Smith, J.C. Ehlert, J. of Electronic Materials, 32, 633 (2003).

13. A.I. D’Souza, M.G. Stapelbroek, P.S. Wijewarnasuriya, R.E. DeWames, D.S. Smith, J.C. Ehlert, SPIE Proceedings Vol. 4820, pg. 389, Intl. Sym. on Optical Science and Tech., 7 - 11 July 2002, Seattle, WA.

14. S.P. Tobin, S. Iwasa, T.J. Tredwell, IEEE Trans. Electron. Devices, ED-27, 43(1980).

15. S. Masterjohn, A.I. D'Souza, L.C. Dawson, P. Dolan, P.S. Wijewarnasuriya, J. Ehlert, SPIE Proceedings Vol. 4820, pg. 368, International Symposium on Optical Science and Technology, 7 - 11 July 2002, Seattle, WA.

16. A.I. D’Souza, L.C. Dawson, S. Marsh, R. Willis, P.S. Wijewarnasuriya, R.E. DeWames, J.M. Arias, J. Bajaj, G. Hildebrandt, F.E. Moore, SPIE Proc. Vol. 4369, p. 157. 